\title{
Eficiência de espécies de adubos verdes sobre a população do nematoide reniforme
}

\author{
Efficiency of green manure species on the population of \\ reniform nematode
}

\author{
Cristiane Gonçalves Gardiano ${ }^{1 *}$; Alaide Aparecida Krzyzanowski²; \\ Otavio Jorge Grigoli Abi Saab ${ }^{3}$
}

\begin{abstract}
Resumo
O objetivo do trabalho foi avaliar o cultivo de plantas melhoradoras de solo na população de Rotylenchulus reniformis em solo naturalmente infestado. Foram avaliadas 6 espécies de plantas de inverno, 13 espécies de verão e um tratamento em pousio, em condições de casa de vegetação. Após 60 dias, o sistema radicular foi coletado e uma amostra de solo correspondente a cada tratamento foi coletada para extração dos juvenis e quantificação da população do nematoide em cada vaso determinando-seo fator de reprodução (FR). O pousio e as espécies de adubos verdes de inverno, exceto a ervilhaca peluda, diminuíram a população de $R$. reniformis, comparando com a testemunha. Em relação às plantas de verão, observou-se que o sorgo 'SI03204' (Sorghum vulgare), o milheto 'BRS1501' (Pennisetum glaucum), a Brachiaria ruziziensis, o capim pé de galinha gigante (Eleusine coracana), o estilosante 'Campo Grande' (Stylosanthes capitata x S. macrocephala), o amendoim 'IAC Tatu ST' (Arachis hypogaea) e a mucuna anã (Mucuna deeringiana) diminuíram a população de $R$. reniformis, quando comparadas à testemunha, podendo ser usadas no manejo deste nematoide.

Palavras-chave: Rotylenchulus reniformis, manejo sustentável, sucessão de culturas, rotação de culturas
\end{abstract}

\begin{abstract}
The objective of this study was to evaluate the growing of soil improving crops on the population of Rotylenchulus reniformis in naturally infested soil. It was evaluated the effect of 6 species of plants as cover crops in winter and 13 summer species and a fallow treatment on the nematode population under greenhouse. After 60 days, the root system was collected. Then, a sample of soil was taken in order to extract juveniles from the soil and quantification the final population of the pathogen in each pot for determining of the reproduction factor (RF). Fallow and all winter species of green manure, except hairy vetch, reduced the population of $R$. reniformis after cultivation in infested soil, in comparison to the control. Regarding summer cover crops, it was observed that sorghum 'SI03204' (Sorghum vulgare), millet 'BRS1501' (Pennisetum glaucum), Brachiaria ruziziensis, finger millet (Eleusine coracana), estylo 'Campo Grande' (Stylosanthes capitata $x$ S. macrocephala), peanut 'IAC Tatu ST' (Arachis hypogaea) and dwarf velvet bean (Mucuna deeringiana) reduced the population of $R$. reniformis, when compared to the control, could be used in the management of this nematode.
\end{abstract}

Key words: Rotylenchulus reniformis, sustainable management, crop succession, crop rotation

\footnotetext{
${ }^{1}$ Discente de Doutorado do Programa de Pós-graduação em Agronomia, Universidade Estadual de Londrina, UEL, Londrina, PR. E-mail: cris_gardiano@yahoo.com.br

${ }^{2}$ Pesquisadora da Área de Proteção de Plantas/Nematologia do Instituto Agronômico do Paraná, IAPAR, Londrina, PR. E-mail: alaidekrzyza@iapar.br

3 Prof. Adjunto do Centro de Ciências Agrárias, Dept ${ }^{\circ}$ de Agronomia, UEL, Londrina, PR. E-mail: abisaab@uel.br

* Autor para correspondência
} 


\section{Introdução}

Rotylenchulus reniformis Linford e Oliveira (1940), conhecido como nematoide reniforme, é um dos principais problemas sanitários para culturas do algodoeiro (Gossypium hirsutum L.) e da soja (Glycine $\max$ L.), por ocasionar perdas em torno de $60 \%$ e 32\%, respectivamente (STARR, 1998; ASMUS; ISEMBERG, 2003; ROBINSON, 2002). Este fitoparasita é um nematoide polífago e se encontra amplamente disseminado nas regiões tropicais e subtropicais (ROBINSON et al., 1997). Assim, o sucesso no manejo deste nematoide em áreas já infestadas depende de um conjunto de medidas associadas (RUANO et al., 1992).

$\mathrm{Na}$ literatura são citados muitos métodos de controle de nematoides, entre eles o pousio, a destruição de plantas infectadas, o uso de material de plantio isento de nematoides, o emprego de métodos para diminuir a disseminação, a consorciação de plantas, rotação de culturas, controle genético, entre outros (TIHOHOD, 1993; FERRAZ et al., 2010). No entanto, os métodos de controle nem sempre são praticados em todas as áreas, pois o manejo eficiente de populações de nematoide requer uma combinação cuidadosa de diferentes táticas de controle economicamente viáveis e exequíveis (THOMASON; CASWELL, 1987).

O emprego de plantas como a aveia (Avena spp. ), o milheto (Pennisetum glaucum (L.) R. Brown), a crotalária (Crotalaria spp.) e a mucuna (Mucuna spp.), entre outras, utilizadas como culturas de cobertura, rotação, sucessão e/ou consorciação com espécies de importância econômica tem reduzido as populações de fitonematoides, pois muitas dessas plantas comportam-se como más hospedeiras, não permitindo a penetração e/ou multiplicação do nematoide em suas raízes, seja produzindo aleloquímicos, seja estimulando o aumento de antagonistas no solo (HALBRENDT, 1996; McSORLEY et al., 1994; RODRIGUEZ-KÁBANA et al., 1998)

A população do nematoide reniforme tende a diminuir na ausência de plantas hospedeiras ou sob condições climáticas desfavoráveis. Assim, a rotação ou sucessão com espécies vegetais não hospedeiras pode constituir uma importante estratégia de manejo de áreas infestadas com este fitoparasita (ASMUS; ISHIMI, 2009; ASMUS; INOMOTO; CARGNIN, 2008). Sob este aspecto, muitos estudos mostraram que algumas plantas, como o centeio (Secale cereale L.) (GUERTAL et al., 1998), a Crotalaria juncea L., Digitaria decumbens Stent (CASWELL; DEFRANK; TANG, 1991), o Tagetes patula L. (KO; SHIMITT, 1996) e o capim rhodes (Cloris gayana Kunth) (CASWELL; DEFRANK; TANG, 1991) diminuíram a densidade populacional de $R$. reniformis.

O objetivo deste trabalho foi avaliar a eficiência de espécies de adubos verdes de inverno e de verão sobre a população de $R$. reniformis, em solo naturalmente infestado.

\section{Material e Métodos}

Foram realizados dois experimentos separadamente, em condições de casa de vegetação, no Instituto Agronômico do Paraná- Iapar, LondrinaPR. No primeiro, foram avaliadas as espécies de inverno no período de 25/06/09 a 25/08/09. As médias de temperatura máxima e mínima neste período foram de $32,7^{\circ} \mathrm{C}$ e $21,0^{\circ} \mathrm{C}$, respectivamente. No segundo, foram avaliadas as espécies de verão, no período de 22/10/09 a 22/12/09, com médias de temperatura máxima e mínima de $36,2^{\circ} \mathrm{C}$ e $22,3^{\circ} \mathrm{C}$, respectivamente.

Foram avaliadas seis espécies de adubos verdes de inverno e 13 espécies de verão, e como testemunha de viabilidade de inóculo foram utilizados a cultivar de algodão 'CD 401' e um tratamento em pousio. O delineamento estatístico utilizado foi o inteiramente casualizado com seis repetições.

Exceto as sementes de estilosante 'Campo Grande', cedidas pela Embrapa Centro Oeste, Dourados, MS, as demais sementes utilizadas foram cedidas pelo Iapar/Londrina, PR. As espécies vegetais utilizadas estão descritas na Tabela 1. 
Tabela 1. Espécies de adubos verde utilizadas no experimento.

\begin{tabular}{|c|c|c|}
\hline & Nome comum & Nome científico \\
\hline \multirow{6}{*}{ 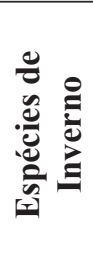 } & Aveia branca 'IPR 126' & Avena sativa $\mathrm{L}$. \\
\hline & Aveia preta 'IAPAR 61' & Avena strigosa Schieb \\
\hline & Centeio 'IPR 89' & Secale cereale $\mathrm{L}$. \\
\hline & Ervilhaca peluda & Vicia villosa Roth \\
\hline & Tremoço branco & Lupinus albus $\mathrm{L}$. \\
\hline & Triticale 'IPR 111' & Triticosecale rimpaui Wittmack \\
\hline \multirow{13}{*}{ 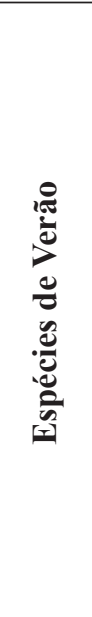 } & Amendoim 'IAC Tatu ST' & Arachis hypogaea $\mathrm{L}$. \\
\hline & Brachiaria ruziziensis & Brachiaria ruziziensis Stapf. \\
\hline & Capim moha & Setaria italica (L.) Beauv. \\
\hline & Capim pé de galinha gigante & Eleusine coracana (L.) Gaertn \\
\hline & Estilosante 'Campo Grande' & $\begin{array}{l}\text { Stylosanthes capitata Vog. + S. macrocephala M. B. Ferreira } \\
\text { e S. Costa }\end{array}$ \\
\hline & Feijão caupi & Vigna unguiculata $(\mathrm{L}$.$) Walp$ \\
\hline & Feijão bravo do Ceará & Canavalia brasiliensis Mart e Benth \\
\hline & Guandu anão 'IAPAR 43' & Cajanus cajan (L.) Millsp \\
\hline & Guandu fava larga & Cajanus cajan (L.) Millsp \\
\hline & Milheto 'BRS 1501' & Pennisetum glaucum (L.) R. Brown \\
\hline & Milho 'IPR 115’ & Zea mays L. \\
\hline & Mucuna anã & Mucuna deeringiana Steph e Bart \\
\hline & Sorgo 'SI 03204' & Sorghum vulgare Pers \\
\hline
\end{tabular}

Fonte: Elaboração dos autores.

Como substrato, utilizou-se solo naturalmente infestado com $R$. reniformis, coletado na área experimental localizada na estação de pesquisa do Iapar em Londrina, PR. Primeiramente, foram coletadas amostras na área e feita uma análise prévia para identificação dos nematoides presentes, constatando-se a presença de Rotylenchulus reniformis, única espécie daninha.. Este solo foi homogeneizado e distribuído em vasos de plástico com capacidade de $3 \mathrm{~kg}$, identificados e adubados individualmente com a dosagem de $0,46 \mathrm{~g}$ de $\mathrm{KCL}$, 4,79 $\mathrm{g}$ de superfosfato simples e $0,5 \mathrm{~g}$ de ureia por vaso. Em seguida, transplantou-se uma muda de algodão 'CD401' com 20 dias de idade, que permaneceu por 30 dias para multiplicaro inóculo.

Após esse período, foi retirada separadamente uma amostra de $50 \mathrm{~cm}^{3}$ de solo de cada vaso para a extração dos nematoides do solo por meio da técnica do funil de Baermann modificado (RUANO; BRITO, 1990). Em seguida, fez-se a contagem para determinação da população inicial (Pi) do nematoide em cada vaso.
As plântulas das espécies de adubo verde de inverno e verão foram obtidas em copos de plástico descartáveis $(150 \mathrm{~mL})$, nos quais foi colocado o substrato Plantmax ${ }^{\circledR}$ e depositadas três sementes por copo. Após 20-30 dias, foi transplantada uma plântula em cada vaso, em seus respectivos tratamentos.

Após 60 dias, coletou-se novamente uma amostra de $50 \mathrm{~cm}^{3}$ de solo de cada vaso após a retirada do sistema radicular, identificada de acordo com seu respectivo tratamento, para montagem do funil de Baermann e extração dos juvenis. Em seguida, efetuou-se a contagem da população final (Pf) em microscópio de luz, determinando-se os fatores de reprodução $(\mathrm{FR}=\mathrm{Pf} / \mathrm{Pi})$. Foram consideradas plantas resistentes aquelas com $\mathrm{FR}<1$ e suscetíveis quando FR $>1$ (OOSTENBRINK, 1966).

Os dados obtidos foram submetidos à análise de variância e teste de médias Scott-Knot, com o programa SASM-Agri (CANTERI et al., 2001). Os valores referentes ao peso da raiz fresca das 
espécies de inverno e de verão foram transformados para $\sqrt{ }(\mathrm{x}+0,1)$. Os dados referentes à $\mathrm{Pi}$ e $\mathrm{Pf}$, das espécies de inverno foram transformados para $\sqrt{ } \mathrm{x}$. Os dados referentes à $\mathrm{Pf}$ do nematoide das espécies de verão foram transformados para $\log _{10}(\mathrm{x})$. E os dados de FR das espécies de inverno e verão, para $\sqrt{ }(x+0,01)$ e $\sqrt{ }(x+1)$, respectivamente. Em seguida, as médias foram submetidas à análise de variância $\mathrm{e}$ comparadas pelo teste de agrupamento Scott-Knott, em nível de 5\% de probabilidade.

\section{Resultados e Discussão}

As espécies de inverno avaliadas, exceto a ervilhaca peluda, diminuíram a população de $R$. reniformis no solo após o cultivo por 60 dias (FR $<1)$, comparando com a testemunha $(\mathrm{P} \leq 0,05)$ (Tabela 2).

Tabela 2. Peso fresco da raiz, populações de juvenis (J2) de Rotylenchulus reniformis no solo, antes e após o cultivo com as espécies de adubos verde de inverno em solo naturalmente infestado e fator de reprodução (FR).

\begin{tabular}{lccccccc}
\hline \multicolumn{1}{c}{ Tratamento } & $\begin{array}{c}\text { Peso Fresco da } \\
\text { Raiz (g) }\end{array}$ & \multicolumn{2}{c}{$\begin{array}{c}\text { População Inicial } \\
(\mathbf{J 2} \text { no solo) }\end{array}$} & $\begin{array}{c}\text { População final } \\
(\mathbf{J 2} \text { no solo) }\end{array}$ & FR $^{\mathbf{2}}$ \\
\hline Centeio 'IPR 89' & 2,45 & a & 22306,67 & a & 5946,67 & a & 0,27 \\
Pousio & --- & & 19826,67 & a & 4000,00 & a & 0,20 \\
Triticale 'IPR 111' & 2,10 & a & 17240,00 & a & 5953,33 & a & 0,34 \\
Ervilhaca peluda & 0,44 & b & 17093,33 & a & 21106,67 & b & 1,23 \\
Aveia preta 'IAPAR 61' & 2,37 & a & 14933,33 & b & 5213,33 & a & 0,35 \\
Aveia branca 'IPR 126' & 1,97 & a & 12453,33 & b & 4713,33 & a & 0,38 \\
Tremoço branco & 3,98 & a & 9973,33 & b & 5980,00 & a & 0,60 \\
Algodão 'CD 401' & 0,91 & b & 18080,00 & a & 32513,33 & b & 1,80 \\
\hline CV & $\mathbf{2 5 , 7 8}$ & & $\mathbf{1 6 , 8 8}$ & & $\mathbf{2 8 , 3 3}$ & & $\mathbf{3 2 , 1 3}$ \\
\hline
\end{tabular}

Médias originais obtidas de seis repetições. Médias seguidas de mesma letra não diferem entre si, a 5\% de probabilidade, pelo teste de Scott-Knott. Valores transformados: ${ }^{1-}$ Peso fresco da raiz, para $\sqrt{ }(\mathrm{x}+0,1)$; ${ }^{2-}$ Pop inicial e final, para $\sqrt{ } \mathrm{x} ;{ }^{3-} \mathrm{FR}=\mathrm{Pf} / \mathrm{Pi}$; Valores transformados para $\sqrt{ }(\mathrm{x}+0,01)$. FR $<1,0(\mathrm{R}=$ Resistente $)$ e $\mathrm{FR}>=1,0(\mathrm{~S}=$ Suscetível $)($ OOSTENBRINK, 1966).

Fonte: Elaboração dos autores.

Os dados obtidos neste estudo confirmam o trabalho feito por Jones, Lawrence e Lawrence (2006), em que observaram que os tratamentos com o cultivo de aveia preta, centeio, tremoço branco e o pousio diminuíram a densidade populacional de $R$. reniformis. No entanto, esses autores verificaram também que a ervilhaca peluda após 120 dias do plantio possibilitou uma diminuição na população do nematoide, quando avaliadas em condições de campo com $\mathrm{FR}<1$. No entanto, em testes realizados em condições de casa de vegetação, a ervilhaca apresentou FR > 1após 60 dias da inoculação, confirmando o ocorrido no presente trabalho. De acordo com estes resultados, supõe-se que o efeito dessa planta na diminuição da população do $R$. reniformis possa ser influenciado por outros fatores além da microbiota do solo, como as condições ambientais, uma vez que, no presente trabalho, mesmo sendo solo naturalmente infestado, não ocorreu diminuição da população de $R$. reniformis.

Com relação às espécies vegetais de verão, cinco espécies de gramíneas (Brachiaria ruziziensis, sorgo 'SI 03204', milheto 'BRS1501', capim pé de galinha gigante e milho 'IPR115') e três de leguminosas (estilosante 'Campo Grande', amendoim 'IAC Tatu ST' e mucuna anã) apresentaram FR $<1$, comportando-se como resistentes, promovendo diminuição na $\mathrm{Pf}$ de $R$. 
reniformis $(\mathrm{P} \leq 0,05)$ (Tabela 3$)$. No entanto, o capim moha, o feijão bravo do Ceará e o tratamento pousio apresentaram FR $>1$, comportando-se como hospedeiras desse nematoide (Tabela 3 ). As demais espécies não diferiram da testemunha $(\mathrm{P} \leq 0,05)$, quanto à Pf e apresentaram FR $>1$, comportandose como suscetíveis (Tabela 3).

Tabela 3. Peso fresco da raiz, populações de juvenis (J2) de Rotylenchulus reniformis no solo, antes e após o cultivo com as espécies de adubos verde de verão em solo naturalmente infestado e fator de reprodução (FR).

\begin{tabular}{lrrrrrrr}
\hline \multicolumn{1}{c}{ Tratamento } & $\begin{array}{c}\text { Peso Fresco } \\
\text { da Raiz (g) }\end{array}$ & $\begin{array}{c}\text { População Inicial } \\
\text { (J2 no solo) }\end{array}$ & $\begin{array}{c}\text { População final } \\
\text { (J2 no solo) })^{\mathbf{2}}\end{array}$ & FR $^{\mathbf{3}}$ \\
\hline Brachiaria ruziziensis & 21,22 & $\mathrm{a}$ & 3700,00 & $\mathrm{a}$ & 1450,00 & $\mathrm{~b}$ & 0,40 \\
Sorgo 'SI 03204' & 19,08 & $\mathrm{a}$ & 3375,00 & $\mathrm{a}$ & 2475,00 & $\mathrm{~b}$ & 0,73 \\
Milheto 'BRS 1501' & 16,76 & $\mathrm{~b}$ & 2958,33 & $\mathrm{a}$ & 1466,67 & $\mathrm{~b}$ & 0,50 \\
Guandu anão 'IAPAR 43' & 5,32 & $\mathrm{c}$ & 2925,00 & $\mathrm{a}$ & 253400,00 & $\mathrm{a}$ & 86,63 \\
Estilosante 'Campo Grande' & 0,97 & $\mathrm{~d}$ & 2591,67 & $\mathrm{a}$ & 1758,33 & $\mathrm{~b}$ & 0,68 \\
Guandu fava larga & 11,70 & $\mathrm{~b}$ & 2500,00 & $\mathrm{a}$ & 226733,33 & $\mathrm{a}$ & 90,70 \\
Capim pé de galinha gigante & 26,29 & $\mathrm{a}$ & 2358,33 & $\mathrm{~b}$ & 1241,67 & $\mathrm{~b}$ & 0,53 \\
Amendoim 'IAC Tatu ST' & 3,15 & $\mathrm{c}$ & 2125,00 & $\mathrm{~b}$ & 1350,00 & $\mathrm{~b}$ & 0,64 \\
Pousio & ---- & & 2108,33 & $\mathrm{~b}$ & 2608,33 & $\mathrm{~b}$ & 1,24 \\
Feijão bravo do Ceará & 7,04 & $\mathrm{c}$ & 2108,33 & $\mathrm{~b}$ & 3083,33 & $\mathrm{~b}$ & 1,46 \\
Milho 'IPR 115' & 22,91 & $\mathrm{a}$ & 1783,33 & $\mathrm{~b}$ & 1116,67 & $\mathrm{~b}$ & 0,63 \\
Feijão caupi & 5,05 & $\mathrm{c}$ & 1591,67 & $\mathrm{~b}$ & 294516,67 & $\mathrm{a}$ & 185,03 \\
Mucuna anã & 20,20 & $\mathrm{a}$ & 1558,33 & $\mathrm{~b}$ & 716,67 & $\mathrm{c}$ & 0,46 \\
Capim moha & 4,05 & $\mathrm{c}$ & 1366,67 & $\mathrm{~b}$ & 1725,00 & $\mathrm{~b}$ & 1,26 \\
Algodão 'CD 401' & 4,32 & $\mathrm{c}$ & 2900,00 & $\mathrm{a}$ & 191200,00 & $\mathrm{a}$ & 65,93 \\
\hline CV & $\mathbf{2 0 , 7 1}$ & & $\mathbf{3 5 , 1 5}$ & & $\mathbf{8 , 0 5}$ & \\
\hline
\end{tabular}

Médias originais obtidas de seis repetições. Médias seguidas de mesma letra não diferem entre si, a 5\% de probabilidade, pelo teste de Scott-Knott. Valores transformados: ${ }^{1-P e s o}$ fresco da raiz, para $\sqrt{ }(\mathrm{x}+0,1) ;{ }^{2-} \mathrm{Pop}$ final, para $\log _{10}(\mathrm{X}) .{ }^{3} \mathrm{FR}=\mathrm{Pf} / \mathrm{Pi}$. Valores transformados para $\sqrt{ }(\mathrm{x}+1)$; FR $<1,0(\mathrm{R}=$ Resistente) e $\mathrm{FR}>=1,0(\mathrm{~S}=$ Suscetível) (OOSTENBRINK, 1966).

Fonte: Elaboração dos autores.

A baixa reprodução de $R$. reniformis em espécies de gramíneas, também já foi relatado por Asmus (2005) e Asmus, Inomoto e Cargnin (2008) em seus trabalhos onde também encontraram FR $<1$ nas espécies vegetais sorgo, milheto, capim pé de galinha gigante e aveias branca, preta e amarela, o que demonstra que as espécies de gramíneas podem ser, de modo geral, consideradas opções para serem utilizadas em rotação ou plantas de cobertura em áreas infestadas com $R$. reniformis.

O milho apresentou $\mathrm{FR}=0,63$, o que demonstrou uma eficiência em baixar a população de $R$. reniformis. Essa espécie também já foi reportada em outros trabalhos como sendo não hospedeira deste nematoide, promovendo diminuição da população do nematoide reniforme quando utilizada em rotação com o algodão (DAVIS; WEBSTER, 2005; WINDHAN; LAWRENCE, 1992). No entanto, de acordo com Asmus e Ferreira (2009), um único ano de rotação com essa cultura não seria o suficiente para reduzir a população do nematoide, abaixo dos níveis de dano, pois, em seus estudos, quando se avaliou o cultivo do algodão no ano seguinte, observou-se aumento da população.

Marwoto (2010), ao avaliar 41 espécies de plantas daninhas em relação à hospedabilidade de $R$. reniformis, encontrou que a maior parte dessas plantas que se comportaram como não hospedeiras a este nematoide (FR $<1$ ); entre elas, eram monocotiledôneas: Setaria barbata, Eleusine indica 
e Penisetum purpureum, pertencentes ao mesmo gênero de capim moha (Setaria italica), capim pé de galinha gigante (Eleusine coracana) e milheto (Penisetum glaucum) avaliados neste estudo.

Outro exemplo de monocotiledôneas que apresentaram resistência a $R$. reniformis foi o arroz, Araujo Filho, Machado e Ferraz (2010) avaliaram 14 cultivares de arroz em relação a hospedabilidade ao nematoide reniforme e verificaram que todas as cultivares foram resistentes com médias de fator de reprodução variando de 0,01 a 0,29 .

Entre as leguminosas estudadas, apenas o estilosante, o amendoim e a mucuna anã se comportaram como não hospedeiras de $R$. reniformis, promovendo diminuição na população deste nematoide. A mucuna anã já foi relatada como não hospedeira do Heterodera glycines, Meloidogyne arenaria (WEAVER; RODRIGUEZ-KÁBANA; CARDEN, 1993), Meloidogyne incognita, M. javanica e M. exigua (FERRAZ et al., 2003), tendo na sua parte aérea os compostos L-Dopa, triacontil, tetracosanato, 1-triacontanol que apresentam atividade nematicida, e além desses compostos podem conter ainda fatores antinutricionais, como fenóis e taninos (FERRAZ et al., 2010).

De acordo com os resultados observados neste estudo e com trabalhos anteriores, a maioria das espécies testadas e classificadas como resistentes são monocotiledôneas e, principalmente, as gramíneas; assim, devem ser as principais opções a serem utilizadas como rotação ou plantas de cobertura em áreas infestadas por $R$. reniformis (ASMUS; INOMOTO; CARGNIN, 2008; ASMUS, 2005). O efeito antagônico da maioria das gramíneas a fitonematoides pode estar associado à presença do composto piracotecol, que apresenta atividade nematicida (GNANAPRAGASAM, 1981; GOMMERS, 1981; ALAM et al., 1979 appud FERRAZ et al., 2010). Outro relato é que em algumas espécies e cultivares de gramíneas, a quantidade de flavonas e catecol, presente nas raízes, apresenta correlação com o grau de infecção nos tecidos dessas plantas por Radopholus similis (SARAH et al., 1997 appud FERRAZ et al., 2010).

Outro relato importante neste estudo foi a alta taxa de reprodução de $R$. reniformis em algumas espécies vegetais como guandu anão 'IAPAR43', guandu fava larga e feijão caupi que apresentaram $\mathrm{FR}=86,63$, $\mathrm{FR}=90,69$ e $\mathrm{FR}=185,03$, respectivamente, ambos acima da testemunha algodão que apresentou FR = 72,93 (Tabela 3). A alta reprodução de $R$. reniformis em plantas de guandu também foi relatada por outros autores em avaliações de hospedabilidade (GARDIANO; KRZYZANOWSKI; SAAB, 2012; ARAUJO FILHO et al., 2010). No entanto, Thakar e Yadav (1986) relataram que há uma variação entre cultivares de guandu quanto à resistência a $R$. reniformis, podendo este comportamento estar relacionado ao conteúdo de fenóis em cada genótipo, visto que variedades resistentes a $R$. reniformis apresentaram maiores teores de fenóis em seus tecidos do que variedades suscetíveis.

O feijão caupi, já foi relatado como bom hospedeiro deste nematoide em trabalhos feitos anteriormente por Ponte (1985) e Gardiano, Krzyzanowski e Saab (2012), sendo considerado também como padrão de suscetibilidade no trabalho de Inserra, Dunn e Vovlas (1994), o que justifica seu FR $=185,03$, superior ao da testemunha (FR = 65,93 ) (Tabela 3) no presente estudo.

O uso de culturas de cobertura é uma alternativa para o manejo do nematoide reniforme, sendo que a supressão pode ser devida a essas plantas serem más hospedeiras ou produzirem compostos químicos alelopáticos, ou então favorecerem o aumento da população de microrganismos antagonistas devido ao aumento da matéria orgânica, favorecendo a diversidade da microflora (MULLER; GOOCH, 1982; McSORLEY et al., 1994).

Apesar de a eficiência no uso dessas espécies de plantas de adubos verde e de cobertura ter sido demonstrada nesse estudo e em trabalhos anteriores (DAVIS et al., 2003; JONES; LAWRENCE; LAWRENCE, 2006; ASMUS; RICHETTI, 2010), 
informações envolvendo espécies vegetais para manejo deste nematoide em esquemas de rotação ainda são escassas. Sendo assim, estudos desta natureza devem ser propostos, visto este nematoide apresentar risco para culturas de importância econômica.

Dessa forma conclui-se que as espécies aveia branca 'IPR126', aveia preta 'IAPAR61', triticale 'IPR111', Centeio 'IPR89', tremoço branco, sorgo 'SI03204', milheto 'BRS1501', B. ruziziensis, capim pé de galinha gigante, estilosante 'Campo Grande', amendoim 'IAC Tatu ST' e mucuna anã apresentam potencial para serem utilizadas no manejo de $R$. reniformis, pois promoveram diminuição da população deste nematoide.

\section{Agradecimentos}

Ao Iapar por permitir o desenvolvimento do trabalho em suas instalações; à Universidade Estadual de Londrina e à Coordenação de Aperfeiçoamento de Pessoal de Nível Superior (CAPES), pela concessão da bolsa de doutorado.

\section{Referências}

ARAUJO FILHO, J. V.; INOMOTO, M. M.; GODOY, R.; FERRAZ, L. C. C. B. Reação de linhagens de feijãoguandu a Rotylenchulus reniformis e Pratylenchus zeae. Nematologia Brasileira, Campinas, v. 34, n. 4, p. 204210, 2010.

ARAUJO FILHO, J. V.; MACHADO, A. C. Z.; FERRAZ, L. C. C. B. Host status of some selected brazilian upland rice cultivars to Meloidogyne incognita race 4 and Rotylenchulus reniformis. Nematology, Brill Academic Publishers, Holanda, v. 12, n. 6, p. 929-934, 2010.

ASMUS, G. L. Reação de algumas culturas de cobertura utilizadas no sistema plantio direto ao nematoide reniforme. Dourados: Embrapa Agropecuária Oeste, 2005. 4 p. (Embrapa Agropecuária Centro Oeste. Comunicado técnico, 99).

ASMUS, G. L.; FERREIRA, A. C. B. Manejo do nematóide reniforme através da rotação anual de culturas. In: CONGRESSO BRASILEIRO DO ALGODÃO, 7., 2009, Foz do Iguaçu. Anais... Campina Grande: Embrapa Algodão, 2009. p. 1709-1715. CD-ROM.
ASMUS, G. L.; INOMOTO, M. M.; CARGNIN, R. A. Cover crops for reniform nematode suppression in cotton: greenhouse and field evaluations. Tropical Plant Pathology, Brasília, v. 33, n. 2, p.85-89, 2008.

ASMUS, G. L.; ISEMBERG, K. Danos em algodoeiro associados ao nematoide reniforme Rotylenchulus reniformis em Mato Grosso do Sul. In: CONGRESSO BRASILEIRO DE ALGODÃO, 4., 2003, Goiânia. Anais... Goiânia: Embrapa Algodão, 2003. p. 210. CDROM.

ASMUS, G. L.; ISHIMI, C. M. Flutuação populacional de Rotylenchulus reniformis em solo cultivado com algodoeiro. Pesquisa Agropecuária Brasileira, Brasília, v. 44, n. 1, p. 51-57, 2009.

ASMUS, G. L.; RICHETTI, A. Rotação de culturas para o manejo do nematoide reniforme em algodoeiro. Dourados: Embrapa Agropecuária Oeste, 2010. 26 p. (Embrapa Agropecuária Oeste. Boletim de Pesquisa e Desenvolvimento, n. 55).

CANTERI, M. G.; ALTHAUS, R. A.; VIRGENS FILHO, J. S. das; GIGLIOTI, E. A.; GODOY, C. V. SASM Agri: sistema para análise e separação de médias em experimentos agrícolas pelos métodos Scott-Knott, Tukey e Duncan. Revista Brasileira de Agrocomputação, Londrina, v. 1, n. 2, p. 18-24, 2001.

CASWELL, E. P.; DEFRANK, J.; APT, W. J.; TANG, C. S. Influence of nonhost plants on population decline of Rotylenchulus reniformis. Journal of Nematology, Lakeland, v. 23, n. 1, p. 91-98, 1991.

DAVIS, R. F.; KOENNING, S. R.; KEMERAIT, R. C.; CUMMINGS, T. D.; SHURLEY, W. D. Rotylenchulus reniformis management in cotton with crop rotation. Journal of Nematology, Lakeland, v. 35, n. 1, p. 58-64, 2003.

DAVIS, R. F.; WEBSTER, T. M. Relative host status of selected weeds and crops for Meloidogyne incognita and Rotylenchulus reniformis. Journal Cotton Science, Baton Rouge, v. 9, p. 41-46, 2005.

FERRAZ, S.; FREITAS, L. G. de; LOPES, E. A.; DIASARIEIRA, C. R. Manejo sustentável de fitonematoide. Viçosa: Editora UFV, 2010. 306 p.

FERRAZ, S.; LOPES, E. A.; FERREIRA, P. A.; AMORA, D. X.; FREITAS, C. F.; CAMPOS, A. V. S. Efeito do cultivo de duas espécies de Mucuna sobre a população de Meloidogyne exigua, M. incognita e $M$. javanica. Nematologia Brasileira, Piracicaba, v. 27, n. 2, p. 236-237, 2003. 
GARDIANO, C. G.; KRZYZANOWSKI, A. A.; SAAB, O. J. G. A. Hospedabilidade de plantas melhoradoras de solo à Rotylenchulus reniformis Linford e Oliveira (1940). Arquivos do Instituto Biológico, São Paulo, v. 79, n. 2 , p. $313-317,2012$.

GNANAPRAGASAM, N. C. The influence of cultivating Eragrostis curvula in nematode infested soils, on the subsequent build-up of populations in replanted tea. Tea Quarterly, Sri Lanka, v. 50, n. 4, p. 160-162, 1981.

GOMMERS, F. J. Biochemical interactions between nematodes and plants and their relevance to control. Helminthological Abstracts, Series B, Plant Nematology, California, v. 50, n. 1, p. 9-24, 1981.

GUERTAL, E. A.; SIKORA, E. J.; HAGAN, A. K.; RODRIGUEZ-KÁBANA, R. Effect of winter cover crops on populations of southern root-knot and reniform nematodes. Agriculture, Ecossystem and Environment, Oxford, v. 70, n. 1, p. 1-6, 1998.

HALBRENDT, J. M. Allelopathy in the management of plant-parasitic nematodes. Journal of Nematology, Lakeland, v. 28, n. 1, p. 8-14, 1996.

INSERRA, R. N.; DUNN, R. A.; VOVLAS, N. Host response of ornamental palms to Rotylenchulus reniformis. Journal of Nematology, Lakeland, v. 26, n. 4S, p. 737-743, 1994.

JONES, J. R.; LAWRENCE, K. S.; LAWRENCE, G. W. Evaluation of winter cover crops in cotton cropping for management of Rotylenchulus reniformis. Nematropica, Florida, v. 36, n. 1, p. 53-66, 2006.

KO, M. P.; SCHIMITT, D. P. Changes in plant-parasitic nematode populations in pineapple fields following intercycle cover crops. Journal of Nematology, Lakeland, v. 28, n. 4, p. 546-556, 1996.

MARWOTO, B. Study on host range of reniform nematode (Rotylenchulus reniformis Linford \& Oliveira). Indonesian Journal of Agriculture, Indonesia, v. 3, n. 1, p. 26-31, 2010.

McSORLEY, R.; DICKSON, D. W.; BRITO, J. A. de; HOCHMUTH, R. C. Tropical rotation crops influence nematode densities and vegetable yields. Journal of Nematology, Lakeland, v. 26, n. 3, p. 308-314, 1994.

MULLER, R.; GOOCH, P. S. Organic amendments in nematode control: an examination of the literature. Nematropica, Florida, v. 27, n. 2, p. 127-180, 1982.

OOSTENBRINK, M. Major characteristic of the relation between nematodes and plants. Mededelingen Van De Landbouwhogeschool, Wageningen, v. 66, n. 4, p. 3-46, 1966.
PONTE, J. J. Os nematoides do caupi e sua importância. Nematologia Brasileira, Brasília, v. 11, p. 38-41, 1985.

ROBINSON, A. F. Reniform nematodes: Rotylenchulus species. In: STARR, J. L.; COOK, R.; BRIDGE, J. (Ed.). Plant resistance to parasitic nematodes. Wallingford: CAB International Publishing. 2002. p. 153-174.

ROBINSON, A. F.; INSERRA, R. N.; CASWELLCHEN, E. P.; VOVLAS, N.; TROCCOLI, A. Rotylenchulus species: identification, distribuition, host ranges, and crop plant resistance. Nematropica, Florida, v. 27, n. 2, p. 99-125, 1997.

RODRIGUEZ-KÁBANA， R.; GAZAWAY, W. S.; WEAVER, D. W.; KING, P. S.; WEAVER, C. F. Host suitability of selected tropical legumes and other crops for the reniform nematode, Rotylenchulus reniformis Linford \& Oliveira, 1940. Nematropica, Florida, v. 28, n. 2, p. 195-203, 1998.

RUANO, O.; BRITO, J. A. Utilização de floculantes de argila no método do funil de Baermann. Fitopatologia Brasileira, Brasília, v. 14, p. 12-13, 1990. Suplemento.

RUANO, O.; CARNEIRO, R. G.; BRITO, J. A.; SILVA, J. F. V. Nematoides na cultura do algodoeiro. Informe Agropecuário, Belo Horizonte, v. 16, n. 172, p. 46-48, 1992.

STARR, J. L. Cotton. In: BARKER, K. R.; PEDERSON, G. A.; WINDHAM, G. L. (Ed.). Plant and nematode interactions. Madison: American Society of Agronomy, 1998. cap. 17 , p. 359-379.

THAKAR, N. A.; YADAV, B. S. Role of total phenols in pigeonpea resistance to reniform nematode. Indian Journal of Nematology, Lakeland, v. 16, n. 2, p. 261-263, 1986.

THOMASON, I. J.; CASWELL, E. P. Principles of nematodes control. In: BROWN, R. H.; KERRY, B. R. (Ed.). Principles and practice of nematode control in crops. Australia: Academic Press, 1987. p. 87-130.

TIHOHOD, D. Nematologia agrícola aplicada. Jaboticabal: FUNEP, 1993. 337 p.

WEAVER, D. B.; RODRIGUEZ-KÁBANA, R.; CARDEN, E. L. Velvetbean in rotation with soybean for management of Heterodera glycines and Meloidogyne arenaria. Journal of Nematology, Lakeland, v. 25, n. 4S, p. 809-813, 1993.

WINDHAN, G. L.; LAWRENCE, G. W. Host status of commercial maize hybrids to Rotylenchulus reniformis. Journal of Nematology, Lakeland, v. 24, n. 4S, p. 745$748,1992$. 